\title{
Comparison of subgaleal and subdural closed drainage system in the surgical treatment of chronic subdural hematoma
}

\author{
Sukru Oral, ${ }^{1}$ Resul Emin Borklu, ${ }^{2}$ Ahmet Kucuk, ${ }^{3}$ Halil Ulutabanca, ${ }^{3}$ Ahmet Selcuklu ${ }^{3}$ \\ ${ }^{1}$ Department of Neurosurgery, Kayseri Training and Research Hospital, Kayseri, Turkey \\ ${ }^{2}$ Department of Neurosurgery, Tunceli State Hospital, Tunceli, Turkey \\ ${ }^{3}$ Department of Neurosurgery, Erciyes University Faculty of Medicine, Kayseri, Turkey
}

\begin{abstract}
OBJECTIVE: One or two burr-hole craniostomies with subgaleal or subdural drainage system and irrigation are the most common methods for surgical treatment of CSDH. The aim of this study is to compare the advantages or disadvantages of these techniques used for CSDH.
\end{abstract}

METHODS: Seventy patients were treated by burr-hole subdural drainage or subgaleal drainage system with irrigation. Our patients were classified into two groups according to the operative procedure as follows: Group I, one or two burr- hole craniostomy with subgaleal closed system drainage and irrigation $(n=36)$, Group II, one or two burr-hole craniostomies with subdural closed drainage system and irrigation $(n=38)$. We compared male and female ratios, complication rates, and age distribution between groups.

RESULTS: There was no remarkable difference between recurrence rates of the two groups. Recurrence rate was $6.25 \%$ in Group I and $7.8 \%$ in Group II. Subdural empyema occurred in one of the patients in Group II. Symptomatic pneumocephalus did not develop in patients. Four patients were reoperated for recurrence at an average of 12-20 days after the operation with the same methods.

CONCLUSION: Both of the techniques have a higher cure rate and a lower risk of recurrence. However, subgaleal drainage system is relatively less invasive, safe, and technically easy. So it is applicable for aged and higher risk patients.

Keywords: Chronic subdural hematoma; subdural drainage; subgaleal drainage.

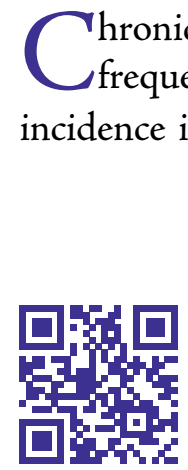

Received: February 04, 2015 Accepted: June 25, 2015 Online: September 26, 2015

Correspondence: Dr. Sukru ORAL. Kayseri Egitim ve Arastirma Hastanesi, Norosirurji Klinigi, Kayseri, Turkey. Tel: +90 352 - 3368884 e-mail: sukruor@yahoo.com

(c) Copyright 2015 by Istanbul Northern Anatolian Association of Public Hospitals - Available online at www.kuzeyklinikleri.com 
The clinical picture of $\mathrm{CSDH}$ varies widely. Common symptoms in the largest series with medical reports on 2300 patients were simple treatment-refractory headache and sensorimotor and neuropsychiatric changes such as amnestic or concentration deficits [2]. Surgical treatment of CSDH in symptomatic patients is still the "gold standard " therapy. The following types of surgical treatment approaches have been used: craniotomy, twist-drill craniostomy, burr-hole drainage with placement of a subdural drain or subgaleal drainage system, percutaneus subdural tapping and endoscopy with variable results.

In this article, we described, and compared the consequences of subdural and subgaleal closed drainage systems.

\section{MATERIALS AND METHODS}

From January 2009 to December 2011, we operated on 78 adult patients with chronic subdural hematomas in the department of Neurosurgery of Erciyes University Faculty of Medicine. All of the patients were treated surgically and analyzed retrospectively. Our patients were classified into two groups according to the operative procedure as follows: Group I, one or two burr- hole craniostomy with subgaleal closed system drainage, and Group II, one or two burr-hole craniostomies with subdural closed drainage system. The choice of the surgical technique was decided randomly by different surgeons. Preoperative clinical laboratory parametres measured included bleeding time, platelet count, prothrombin time, and activated partial thromboplastin time. Our study was approved by institutional ethics committee. In all cases, computed tomography and/or magnetic resonance images was used for diagnosis and postoperative evaluation. We measured thickness of the hematoma using a PACS (Infinitt. Co. Ltd. Seoul, Korea) viewer. Two groups had similar preoperative clinical data.

Standard supplemental statistical methods (mean, standard deviation and percentage) were used to evaluate the results of this study. The normality of the range of the parametric variables was evaluated with the Kolmogrov-Smirnov test. Result were analyzed using Student t-test, Mann-Whitney $\mathrm{U}$ and Wilcoxon Test. P-value of $<0.05$ was considered to be statistically significant.

\section{Surgical techniques}

All operations were performed under sedo-analgesia and general anesthesia. Patients were placed in the supine position with the head elevated and inclined 20-40 degrees towards the contralateral side of the hematoma. As a prophylactic antibiotic, $1 \mathrm{~g}$ cefazolin injection was given before the skin incision.

A skin incision of approximately 4 to $5 \mathrm{~cm}$ in length was made over the maximum thickness of

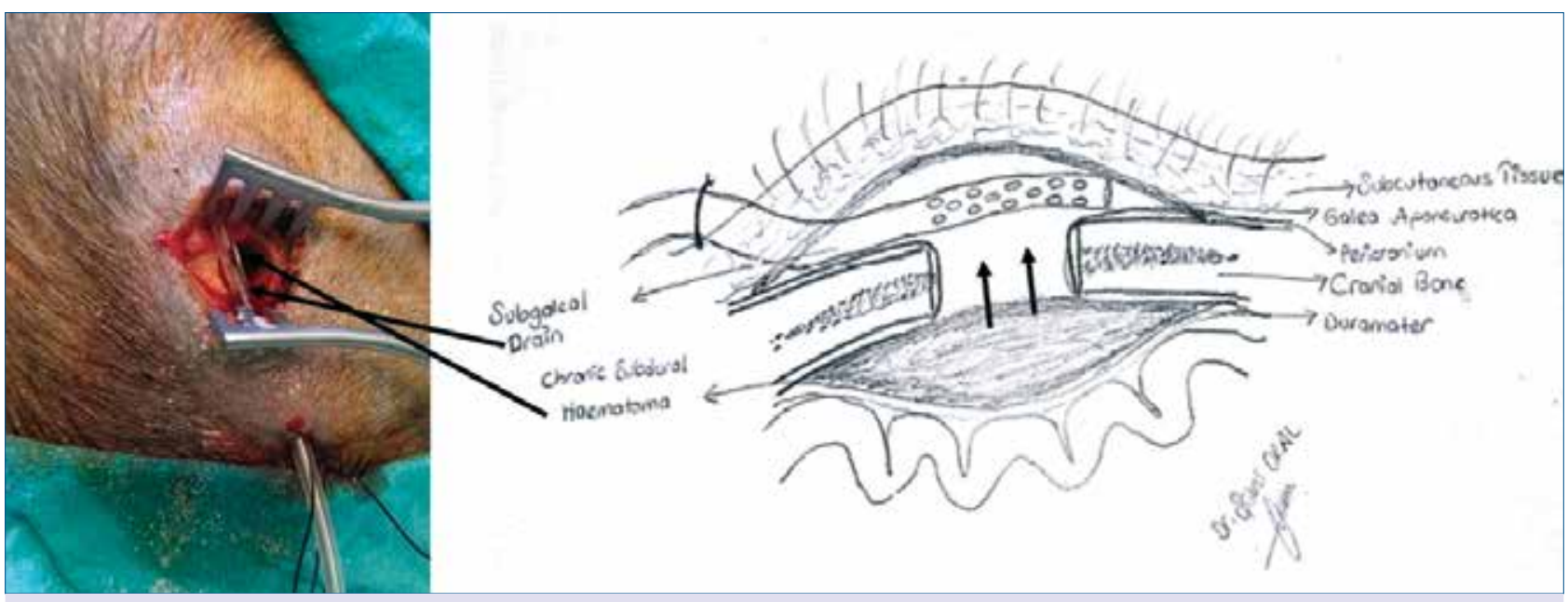

FIGURE 1. Schematic illustration: subgaleal drainage system and single burr-hole craniotomy. 

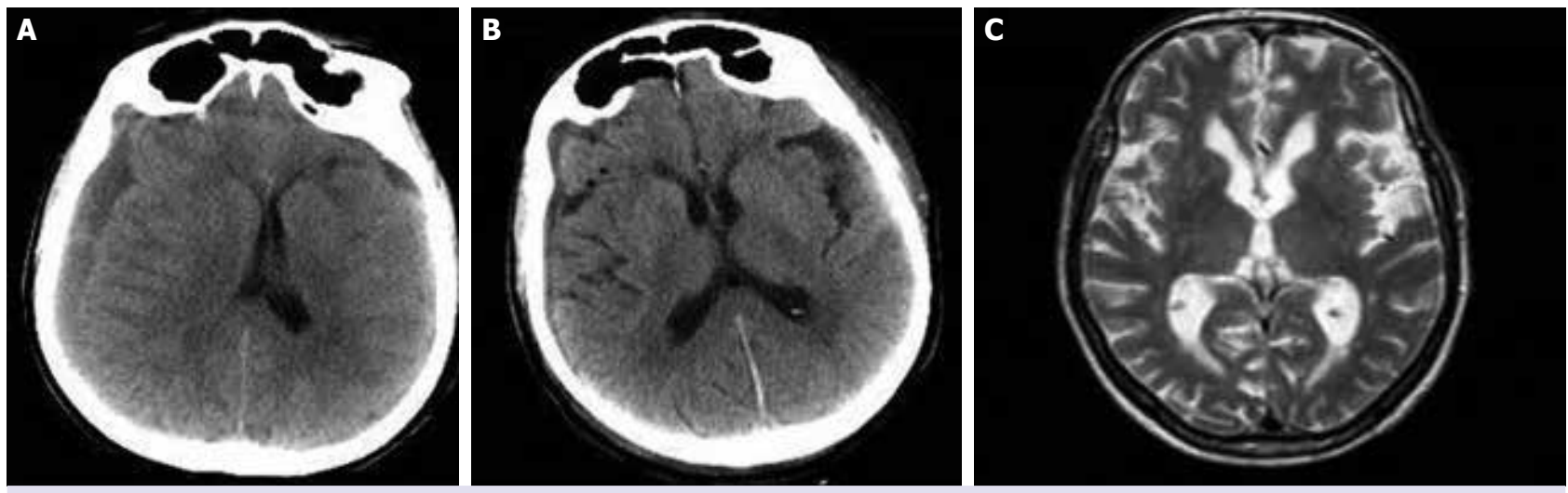

FIGURE 2. Group I and subgaleal drainage system. $72 \mathrm{M} /$ headache, decreased consciousness and hemiparesis (A) Preoperative CT imaging (B) Postoperative second week (C) Postoperative eighth week.

the hematoma, generally the parietal bump. Burrhole craniostomy was performed with the high speed pneumatic drills. The dura mater was incised and bleeding foci were coagulated by bipolar coagulation. The outer and inner membranes of the hematoma were coagulated and incised and hematoma was drained. Then irrigation of the hematoma cavity with sterile saline at $37^{\circ} \mathrm{C}$ was made until clear fluid came out of the burr hole. A $10 \mathrm{~F}$ drainage tube was placed in the subgaleal area and the terminal end of the tube was positioned over the burr hole in Group I (Figure 1). In Group II, the drainage tube was inserted and left in the subdural space. After we closed the incision, the tube was connected to a closed drainage system. The drainage tubes were removed 48-72 hours after the surgery in Groups I and II. Prophylactic antibiotics were used so long as the drainage continued.

\section{RESULTS}

Distribution of males and females in each group was 28:8 in Group I, 29:9 in Group II. The mean ages of the patients in Groups I, and II were 68.1 \pm 14.4 and $66.1 \pm 13.7$ years, respectively. Clinical features of the patients are listed in Table 3. Neurologic performances of the patients were evaluated based on Markwalder Grading Scale (MGS) (Table1).

Fifty-eight $(78.3 \%)$ patients had a history of head trauma and nine patients (11.5\%) were receiving anticoagulant therapy. Four patients had had a ventriculoperitoneal shunt inserted previously for
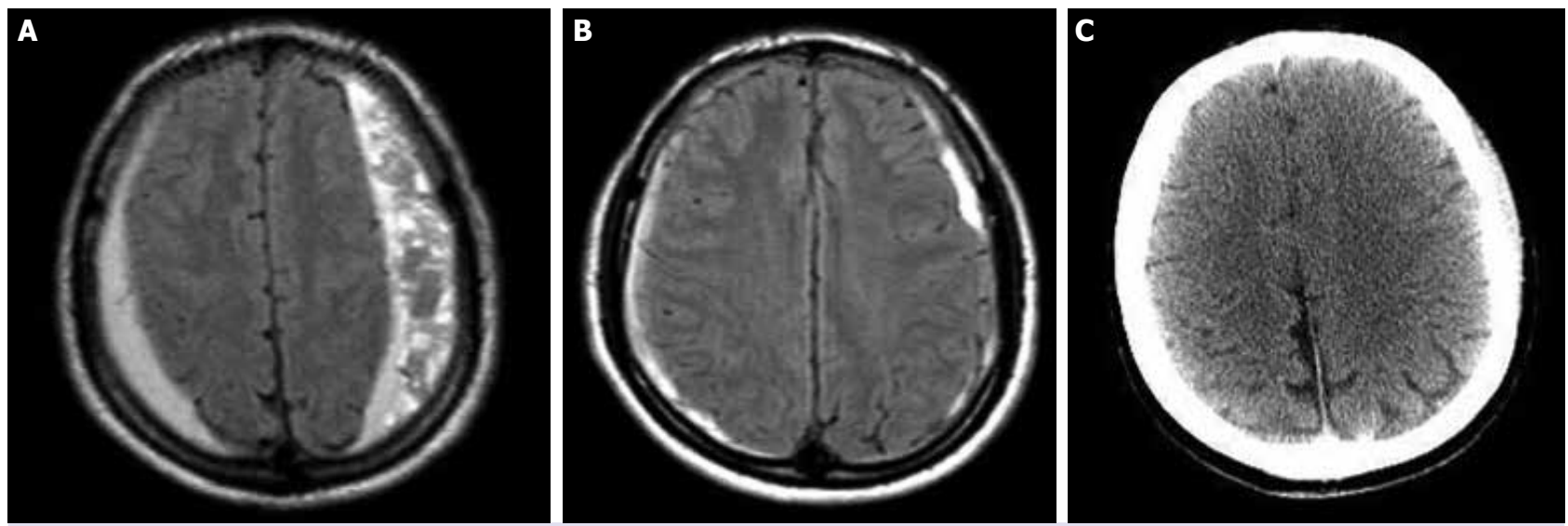

FIGURE 3. Group II and subdural drainage system. 40 M/ headache and vertigo (A) Preoperative MRI (B) Postoperative second week (C) Postoperative eighth week. 


\section{TABLE 1. Markwelder Grade Scale}

\section{Patient neurologically normal}

1 Patient alert and oriented, mild symptoms such as headache, absent or mild neurological deficits such as reflex asymmetry

2 Patient drowsy or disoriented with variable neurological deficits such as hemiparesis

3 Patient stuporous but responding appropriately to noxious stimuli, severe focal signs such as hemiplegia

4 Patient comatose with absent motor responses to painful stimuli, decerebrate or decorticate posturing

hydrocephalus. No precipitating factors could be identified in three patients (Table 2).

Headache was the most common symptom of the patients $(51.3 \%)$. The other symptoms and signs were limb weakness $(51.3 \%)$, decreased consciousness $(20.5 \%)$, seizure (5.1\%), diplopia (1.2\%), vertigo $(11.5 \%)$, and cognitive dysfunction (10.2\%). Hematoma was bilateral in 5 patients $(6.4 \%)$ ( $\mathrm{Ta}$ ble 3). There was no significant difference between groups as for clinical presentations. No statistically significant differences were found regarding the distribution of genders and age groups between groups.

In Group I, 6, and in Group II, 5 patients had grade 3 neurologic performance status based on Markwalder Grading System. At the time of discharge, in Group I, 4, and in Group II, 2 patients had grade 3 neurologic performance status based on Markwalder Grading System. The mean thickness of the hematoma was $2.32 \mathrm{~cm}$ preoperatively in Group I and $2.38 \mathrm{~cm}$ in Group II. Any statistically significant intergroup differences were not found as for the thickness of hematomas ( $p>0.05)$ (Table 4).

All the patients were checked with CT or MRI at the 2.,8., and 12. postoperative weeks. All the patients were discharged between the 2.-5. postopera-

\section{TABLE2. Etiology}

\begin{tabular}{lcc} 
& Group I & Group II \\
\hline Recent head injury & 28 & 30 \\
Shunt related & 2 & 2 \\
Use of anticoagulant drugs & 4 & 5 \\
Unknown & 2 & 1
\end{tabular}

tive days. We followed up the patients for 3 months. The patients have been followed clinically and in the third month control CT, the complete resorption of the hematomas was observed (Figures 2 and 3).

Symptomatic pneumocephalus did not develop in patients. Postoperative complications and outcomes of the patients with $\mathrm{CSDH}$ are shown in Table 5.

A subdural empyema occurred in a patient from Group II who was treated with antibiotics and surgery. Seizures were treated with anticonvulsant drugs (phenytoin or sodium valproate). Recurrence of CSDH was diagnosed according to clinical (aggravation of headeache, change in conciousness, and worsening of preexisting neurological deficit) and/ or radiological criteria (CT scans). Four patients were reoperated for recurrences between 12-20 days after the operation with the same methods. But one patient was reoperated with craniotomy in Group II with the indication of acute subdural hematoma. In the majority of the patients, neurologic status improved after the operation.

\section{DISCUSSION}

Chronic subdural hematoma is seen in geriatric patients, and trauma is the most important reason for CSDH $[1,2,3]$. Treatment options for $\mathrm{CSDH}$ are surgical or nonsurgical therapies. Nonoperative treatment of CSDH consists of the use of steroids (low dose dexamethasone) or mannitol $[4,5,6,7]$. Operative treatment of CSDH in symptomatic patients is yet the gold standard of therapy because it allows urgent decompression of the subdural space [8].

Different operative treatment options have been reported for $\mathrm{CSDH}$, such as twist-drill craniotomy, 


\begin{tabular}{lcc}
\hline TABLE 3. Clinical features & & \\
& Group I & Group II \\
\hline Sex & & \\
$\quad$ Male & 28 & 29 \\
$\quad$ Female & 8 & 9 \\
Median age & $68.1(47-86)$ & $66.1(24-109)$ \\
Presenting symptoms & & \\
$\quad$ Headache & 18 & 20 \\
$\quad$ Decreased consciousness & 7 & 9 \\
$\quad$ Limb weakness & 15 & 13 \\
$\quad$ Seizure & 1 & 3 \\
Diplopia & - & 1 \\
Cognitive dysfunction & 5 & 3 \\
Vertigo & 4 & 5 \\
$\quad$ Unilateral & 34 & 35 \\
Bilateral & 2 & 3 \\
& & \\
\hline
\end{tabular}

small craniotomy and endoscopic removal, large craniotomy and membranectomy or burr-hole craniostomy with or without continuous closed drainage system and continuous subgaleal suction drainage or burr-hole trepanation and a subperiostal drainage system $[9,10,11,12,13,14,15]$.

In this study, we compared two surgical techniques including burr-hole craniostomy, subdural and subgaleal drainage systems. Besides, we discussed the advantages and disadvantages of these techniques.

According to Gazzeri et al. subgaleal closed drainage system has a low rate of recurrence and pneumocephalus [14]. Also, some researchers have reported lower recurrence rates with the use of postoperative drains [16]. In addition, clinical data support the beneficial effects of intraoperative irrigation in order to resolve hematoma and reduce recurrences. Furthermore, use of closed system drainage reduces the risk of recurrence without additional risk of complications [17]. In our study, rate of recurrence was $5.5 \%$ in Group I, and $7.8 \%$ in Group II.

Additionally, rates of pneumocephalus were $43.75 \%$ in Group I and $47.3 \%$ in Group II. Consequently, these techniques have a lower recurrence rate and pneumocephalus.

The craniotomy technique has a low recurrence rate. But it is more invasive and has a greater morbidity and mortality $[2,18]$. Nevertheless, crani-

\begin{tabular}{|c|c|c|c|}
\hline Factors & Group I & Group II & $\mathrm{p}$ \\
\hline Mean age & 68.1 & 66.1 & 0.18 \\
\hline Markwelder's Score & 1.66 & 1.69 & 0.24 \\
\hline Recurrences, n (\%) & $2(5.5)$ & $3(7.8)$ & 0.04 \\
\hline Width of hematoma $(\mathrm{cm})$ & 2.32 & 2.38 & 0.46 \\
\hline Pneumocephalus (\%) & 38.8 & 47.3 & 0.02 \\
\hline
\end{tabular}


TABLE5. Complications and outcomes in Groups I and II

\begin{tabular}{lcc} 
& Group I & Group \\
\hline Pneumocephalus & 14 & 18 \\
Seizures & 1 & 2 \\
Infection & - & 1 \\
Recurrence & 2 & 3
\end{tabular}

otomy as a management of $\mathrm{CSDH}$ has indications as solid hematoma and multiple recurrences [1]. In our study, we used craniotomy for reoperation of one patient who developed a solid hematoma.

Its other surgical complication is postoperative seizures [19]. In our experience, rate of seizures was $2.7-5.2 \%$ in Groups I and II. Seizure rates of $2-19 \%$ have been reported in CSDH patients $[20,21]$.

The other common complication is symptomatic pneumocephalus. This complication has been reported to range from 0 to $10 \%$ in the literature [14, $20,22]$. In the present study this complication did not develop in any patient in Groups I and II. Similarly, Gazzeri et al. did not report any incident of symptomatic pneumocephalus in their series [14].

Subdural empyema occurred in one of the patient who had undergone subdural drainage. The incidence of postoperative empyema has been reported to range between 0 , and 6 percent $[23,24$, 25, 26]. Similarly, Gazzeri et al. and Zumofen et al. reported a very low rate of subdural empyema in association with extracranial placement of the drain $[14,15]$. In addition, Zumofen et al. advocated that the placement of extracranial drainage can reduce the incidence of deep brain infection which might develop as a consequence of surgical treatment of $\mathrm{CSDH}$ [15].

Postoperative acute hemorrhage was reported in some series in association with the use of subdural drain and burr-hole techniques [27]. Gazzeri et al. [14] and Zumofen et al. [15] have signified that the use of a subdural drain with burr-hole may lead to intracerebral hemorrhage. These drainage catheters may penetrate into the brain parenchyma or injure bridging veins $[5,28]$. Placement of subgaleal drain- age tube has not any risk of these complications. In our series, we haven't seen any postoperative acute hemorrhage.

\section{Conclusions}

Subdural and subgaleal drainage system have a higher cure rate and a lower risk of recurrence. However, subgaleal drainage system is relatively less invasive, safe, and technically easy. Regarding safety, we didn't see any subdural empyeme in our patients for whom we used subgaleal drainage system. Since the drainage tube is not in direct contact with the brain tissue and membranes of $\mathrm{CSDH}$ in this method, theoretically, there is no risk of acute cerebral hemorrhage. So subgaleal drainage system is practically applicable for higher risk patients.

Conflict of Interest: No conflict of interest was declared by the authors.

Financial Disclosure: The authors declared that this study has received no financial support.

\section{REFERENCES}

1. Santarius T, Hutchinson PJ. Chronic subdural haematoma: time to rationalize treatment? Br J Neurosurg 2004;18:328-32.

2. Sambasivan M. An overview of chronic subdural hematoma: experience with 2300 cases. Surg Neurol 1997;47:418-22.

3. Mori K, Maeda M. Surgical treatment of chronic subdural hematoma in 500 consecutive cases: clinical characteristics, surgical outcome, complications, and recurrence rate. Neurol Med Chir (Tokyo) 2001;41:371-81.

4. Bender MB, Christoff N. Nonsurgical treatment of subdural hematomas. Arch Neurol 1974;31:73-9.

5. Suzuki K, Sugita K, Akai T, Takahata T, Sonobe M, Takahashi $\mathrm{S}$. Treatment of chronic subdural hematoma by closed-system drainage without irrigation. Surg Neurol 1998;50:231-4.

6. Sun TF, Boet R, Poon WS. Non-surgical primary treatment of chronic subdural haematoma: Preliminary results of using dexamethasone. Br J Neurosurg 2005;19:327-33.

7. Amos OA. Non-operative treatment of chronic subdural hematoma: Case Report Indian. Journal of Neurotrauma (IJNT) 2009;6:1.

8. Weigel R, Krauss JK, Schmiedek P. Concepts of neurosurgical management of chronic subdural haematoma: historical perspectives. Br J Neurosurg 2004;18:8-18.

9. Camel M, Grubb RL Jr. Treatment of chronic subdural hematoma by twist-drill craniotomy with continuous catheter drainage. J Neurosurg 1986;65:183-7. 
10. Rodziewicz GS, Chuang WC. Endoscopic removal of organized chronic subdural hematoma. Surg Neurol 1995;43:569-72.

11. Hamilton MG, Frizzell JB, Tranmer BI. Chronic subdural hematoma: the role for craniotomy reevaluated. Neurosurgery 1993;33:67-72.

12. Markwalder TM. Chronic subdural hematomas: a review. J Neurosurg 1981;54:637-45.

13. Markwalder TM, Seiler RW. Chronic subdural hematomas: to drain or not to drain? Neurosurgery 1985;16:185-8.

14. Gazzeri R, Galarza M, Neroni M, Canova A, Refice GM, Esposito $\mathrm{S}$. Continuous subgaleal suction drainage for the treatment of chronic subdural haematoma. Acta Neurochir (Wien) 2007;149:487-93.

15. Zumofen D, Regli L, Levivier M, Krayenbühl N. Chronic subdural hematomas treated by burr hole trepanation and a subperiostal drainage system. Neurosurgery 2009;64:1116-22.

16. Wakai S, Hashimoto K, Watanabe N, Inoh S, Ochiai C, Nagai $\mathrm{M}$. Efficacy of closed-system drainage in treating chronic subdural hematoma: a prospective comparative study. Neurosurgery. 1990;26:771-3.

17. Weigel R, Schmiedek P, Krauss JK. Outcome of contemporary surgery for chronic subdural haematoma: evidence based review. J Neurol Neurosurg Psychiatry 2003;74:937-43.

18. Jeong CA, Kim TW, Park KH, Chi MP, Kim JO, Kim JC. Retrospective analysis of reoperated patients after chronic subdural hematoma surgery. J Korean Neurosurg Soc 2005;38:116-20.

19. Rubin G, Rappaport ZH. Epilepsy in chronic subdural haematoma. Acta Neurochir (Wien) 1993;123:39-42.
20. Kravtchouk AD, Likhterman LB, Potapov AA, El-Kadi H. Postoperative complications of chronic subdural hematomas: prevention and treatment. Neurosurg Clin N Am 2000;11:547-52.

21. Leung GK, Fan JK, Tam MC, Fan YW. Surgical complications of chronic subdural haematoma: A 5-year audit. Ann. Coll. Surg. H.K 2001;5:99-103.

22. Sharma BS, Tewari MK, Khosla VK, Pathak A, Kak VK. Tension pneumocephalus following evacuation of chronic subdural haematoma. Br J Neurosurg 1989;3:381-7.

23. Hennig R, Kloster R. Burr hole evacuation of chronic subdural haematomas followed by continuous inflow and outflow irrigation. Acta Neurochir (Wien) 1999;141:171-6.

24. Weir B. Oncotic pressure of subdural fluids. J Neurosurg 1980;53:512-5.

25. Pencalet P. Complications of chronic subdural hematoma in the adult. [Article in French] Neurochirurgie 2001;47:491-4. [Abstract]

26. Rohde V, Graf G, Hassler W. Complications of burr-hole craniostomy and closed-system drainage for chronic subdural hematomas: a retrospective analysis of 376 patients. Neurosurg Rev 2002;25:89-94.

27. Weisse A, Berney J. Chronic subdural haematomas. Results of a closed drainage method in adults. Acta Neurochir (Wien) 1994;127:37-40.

28. Nakajima H, Yasui T, Nishikawa M, Kishi H, Kan M. The role of postoperative patient posture in the recurrence of chronic subdural hematoma: a prospective randomized trial. Surg Neurol 2002;58:385-7. 